\title{
QoS Analysis on OSPFv3 And RIPng Using GRE Tunneling on IPv6 Integrated Ipv4 Network
}

\author{
Indra Warman, Alex Franozal \\ Institut Teknologi Padang, Informatics Engineering Study Program, Padang, Indonesia
}

\begin{abstract}
Every year, the availability of public IPv4 addresses is running low. However, the IETF (Internet Engineering Task Force) has set a new addressing standard called IPv6 (Internet Protocol version 6). IPv6 implementations can not be immediately performed on all end-to-end lines, a transition phase is required, one of which is the GRE tunneling method. IPv6 has some differences with IPv4, then in line with the presence of IPv6 will required the protocol that compatible with IPv6, among which is the routing protocol. Some dynamic routing protocols are created to support and can run on IPv6 such as RIPng and OSPFv3. Aim of this study is examines OSPFv3 routing protocols and RIPng routing protocols in terms of Quality of Service (QoS). The test is done by using seven routers with three scenarios in each routing protocol, ie scenario with best path active condition, when the network changes in other words best path down, and the scenario with best path condition has changed and the network has returned to normal. Testing is done by testing QoS parameters (delay, packet loss, and throughput) when the client computer downloads files with the iso extension from the server. Downloaded files have different sizes, from 100 Mega Byte to 1 Giga Byte. From the results obtained that OSPFv3 provides better QoS (delay, packet loss, and throughput) than RIPng on integrated IPv6 network using GRE tunnel and can be a reference when going to transition from IPv4 to IPv6 using GRE Tunnel.
\end{abstract}

\section{Introduction}

Based on data from LACNICLabs Opendata (opendata.labs.lacnic.net), from year to year the availability of public IPv4 addresses is descreasing, recorded on May 23, 2014 available 19,435,520 IPv4 for regional APNIC, but on May 24, 2017 the remaining $6,442,496$ IPv4. However, the IETF (Internet Engineering Task Force) has set a new addressing standard called IPv6 (Internet Protocol version 6). There are some differences between IPv6 and IPv4 where IPv6 also required supporting protocols, one of them is routing protocol. Some dynamic routing protocols are created to support and can run on IPv6 such as RIPng and OSPFv3.

However, the outline of IPv6 implementation can not be immediately done in all end-to-end lines. The transition mechanism has been included in the IPv6 design, one of which is the tunneling method. Among the IPv6 over IPv4 tunneling methods that can be used are GRE (Generic Routing Encapsulation) tunneling. One of objectives of technology utilization is to produce services of good quality, as well as transition from IPv4 to IPv6. Proper application of routing protocols can help improve performance and provide good network service quality.

Researches related to this topic have been widely practiced. Performance testing of RIPng, OSPFv3 and EIGRP routing protocols in the IPv6 network using
GNS3 based on throughput, jitter and packet loss parameters which then obtained various results in experiments for each routing protocol and combination of routing protocols. RIPng is better than OSPFv3 and EIGRP in terms of TCP throughput [1]. For UDP packets, the best jitter values are achieved by OSPFv3 and EIGRP, while RIPng-OSPFv3 obtains the highest jitter. RIPng is better in terms of packet loss, while OSPFv3 obtains the highest average packet loss.

Discuss various aspects of IPv4 and IPv6, IPv4 transition technology to IPv6, GRE (Generic Routing Encapsulation) and demonstrate how to configure IPv6 tunneling over IPv4 on routers using GNS3 and Wireshark. GRE tunnel runs well on the simulation and can complete IPv6 packet over IPv4 cloud [2]. Migration techniques from IPv4 network to IPv6 network using dual stack method and tunneling migration technique were simulated using GNS3.

Evaluate the performance of different routing protocols such as RIP and OSPF for IPv6 using the OPNET 14.5 simulator and obtain the result that Packet Delay variations in Video Conferencing, based on jitter parameters, end to end delay, received traffic, and traffic transmitted in voice and object response times and page response time in HTTP performs better in OSPFv3_RIPng (combination of RIPng and OSPFv3) than RIPng or OSPFv3. For real time applications, based on end to end delay parameters, received traffic and traffic sent in Video Conferencing, Response Time in 
Query Database runs better in OSPFv3 than OSPFv3 RIPNG and RIPNG. In the case of the performance of each routing protocol, the overall performance of OSPFv3 is better than RIPng [3].

The transition from IPv4 to the IPv6, and how to reduce the risk of problems that will have a negative impact to the user and the service provider during the transition from IPv4 to the IPv6. Comparison of IPv6 transition mechanisms such as Dual Stack, Automatic Tunneling, and Manually Configured Tunneling in terms of security issues. The study offers a transitional model with Automatic Tunneling as well as Manually Configured Tunneling [4].

Saraj (2015) analyzes the most common tunneling protocols available for configuration in most network devices. The analysis of the research is based on math and deployment in testbed setup on LAN, CAN and MAN only. These activities include the behavior of tunneling protocols with applications that use TCP or UDP over the tunneling protocol. The tunneling protocols studied were ISATAP, GRE 6to4, and 6RD. The performance of the protocol is measured through throughput parameters, end-to-end delay, jitter, RTT and tunnel overhead in terms of TCP and UDP. The results obtained that ISATAP in most parameters have shown better performance than other tunneling protocols. In addition, 6to4 in most cases has shown comparative performance worst against the others. The final conclusion is that if the tunneling mechanism is configured on an end host, not on a network device such as a router, it can provide better performance than the other way around [5].

Juanda (2016) compares the distance vector routing protocol represented by RIPv2 with the protocol link state routing protocol represented by OSPF based on Quality of Service (QoS), the speed of the table update against the use of streaming video, testing when one link or down path to see the speed track displacement process, testing convergence time when the client is in a video stream when the best path suddenly down. The test was performed based on the standard configuration with changes in transmit parameters on the OSPF protocol path of $10 \mathrm{M}$. This research proved that the link state protocol represented by OSPF is better than the distance vector protocol represented by RIP based on jitter, packet loss, and faster parameters in terms of time required for convergence as well as for routing table updates [6].

This research combines the best tunneling method with the most widely used routing protocols obtained from the above research, so it can provide recommendations or references in the selection of the latest and best routing protocols on large-scale network conditions when transition from IPv4 to IPv6.

IP Address version 4 (IPv4) was published in 1981 as informed through RFC791. IPv4 has advantages in terms of ease of network planning and implementation. IPv4 has a 32 bit range. There are about 232 $(4,294,467,295)$ IP addresses that can be used on IPv4. Of these 256 '/ 8' subnet blocks, there are approximately 35,078 blocks used for special needs (already reserved), 16 subnet blocks '/ 8' have been ordered for IP multicast,
16 subnet '/ 8' blocks are used for future use (future use), and several other subnet blocks are also used for special purposes (RFC5737). The IPv4 structure is shown in Fig.1

\begin{tabular}{|c|c|c|c|c|}
\hline 4 & 8 & 16 & & 32 bit \\
\hline Version & IHL & Type of & Tota & Length \\
\hline \multicolumn{3}{|c|}{ Identification } & Flags & $\begin{array}{c}\text { Fragment } \\
\text { offset }\end{array}$ \\
\hline Time & Live & Protocol & \multicolumn{2}{|c|}{ Header Checksum } \\
\hline \multicolumn{5}{|c|}{ Surce Address } \\
\hline \multicolumn{5}{|c|}{ Destination Address } \\
\hline \multicolumn{5}{|c|}{ Option + Padding } \\
\hline \multicolumn{5}{|c|}{ Data } \\
\hline
\end{tabular}

Fig. 1. IPv4 Header

The Internet Engineering Task Force (IETF) initiated the development of IPv4 router protocols in the early 1990s. Here is an overview of major changes to IPv6 from IPv4: expanded address space; autocongiration; simplifying header format; enhanced support for option and extension.

\begin{tabular}{|l|l|l|l|}
\hline bits 4 & \multicolumn{1}{c}{16} & 24 \\
\hline Ver & Traffic Class & \multicolumn{1}{|c|}{ Flow Label } \\
\hline \multicolumn{5}{|c|}{ Payload Length } & Next Header & Hop Limit \\
\hline \multicolumn{4}{|c|}{ Source Address } \\
\hline \multicolumn{3}{|c}{ Destination Address } \\
\hline
\end{tabular}

Fig.2. IPv6 Header

IETF (Internet Engineering Task Force) has formed a team to overcome the interconnection process from IPv4 to IPv6 ie IETF IPNG Transition. The purpose of making this transition mechanism so that IPv6 packets can be passed on an existing IPv4 network or vice versa. During the interconnection process IPv4 and IPv6 networks must be able to communicate with each other IPv4 or IPv6 without reducing its reliability.

The dual stack transition method lies in the internet layer in the TCP / IP protocol that is responsible for the Internet Protocol (IP). Dual stack allows to have IPv4 and IPv6 address simultaneously in an interface.

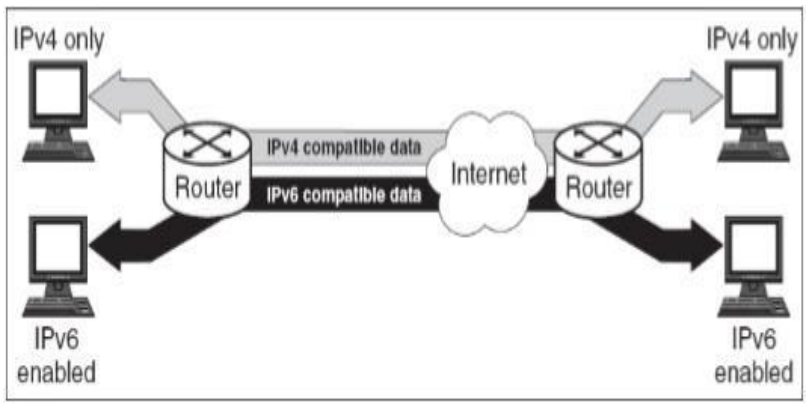

Fig. 3. Dual Stack [7] 
Tunneling is a technique for passing IPv6 packets over an IPv4 network by encapsulating packets. Before being sent, the data packet has a slight alteration or modification, ie the addition of a header to the tunnel. When the data has passed through the tunnel and reached the destination or the end of the tunnel, the header of the data packet will be restored as before. Tunneling method that can be used generally divided into two, namely as follows: Static Point-to-Point IPv6 (Manual Tunnel and GRE Tunnel) and Dynamic Multipoint IPv6 (Automatic 6to4 and Intra-Site Auto Tunneling Addressing Protocol (ISATAP))

GRE is a mechanism to encapsulate network layer protocols through other network layer protocols. The general specification was originally described in RFC1701, and IP packet encapsulation via IP is defined in RFC1702 as a specific implementation of GRE. The GRE specification has been formalized in RFC2784 and is commonly used to encapsulate IPv4 and IPv6 packets in IPv4 packets. RFC2890 extends RFC2784 with key edition (key) and sequence number. The IPv4 47 protocol is used when the GRE packet is encapsulated on IPv4. GRE can encapsulate multicast, broadcast, or other no-IP-type traffic.

Open Shortest Path First version 3 (OSPFv3) is a development routing protocol from OSPFv2 which is included in the interior of Gateway Protocol (IGP). In general the workings between OSPFv2 and OSPFv3 are the same. To achieve full convergence conditions, there are several stages to pass. Similar to OSPFv2, OSPFv3 also has 2 way state. OSPFv3 is capable of running on IPv4 and IPv6 based networks at once. OSPFv3 uses the Djikstra algorithm and uses cost as a metric. The path with the smallest cost value used as the main line. The cost value of OSPFv3 is obtained using the following formula.

$$
\text { Cost }=\frac{\text { Reference Bandwith }(100 \mathrm{MB})}{\text { Interface Bandwith }(\mathrm{MB})}
$$

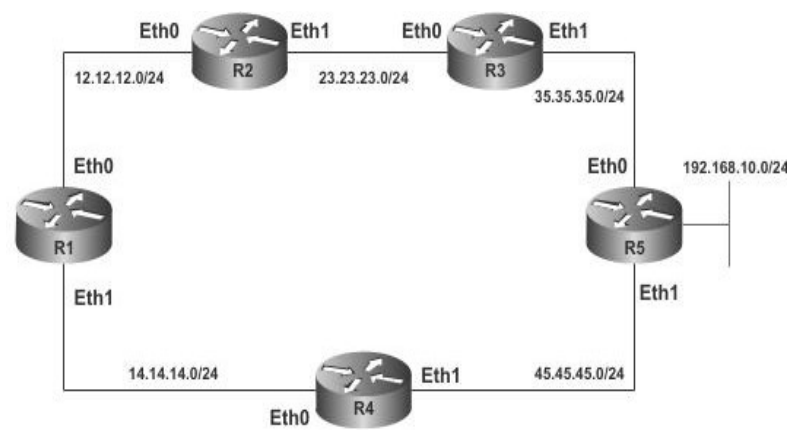

Fig. 4. Hop-Count Concept [8]

RIPng uses the UDP protocol on port 521 to transport, either in sending or receiving datagrams. RIPng is a development of RIPv2. Basically, the workings of RIPng are the same as RIPv2. But one of the fundamental differences is on IPv6 addressing support. RIPng is a distance-vector routing protocol known as the BellmanFord algorithm and entered in the Interior Gate Protocol class developed by IETF. Distance Vector Protocol is a dynamic routing technique that uses distance and direction as the main guide in routing. The distance is the number of routers passed, while the direction in question is the address of the router through. The use of the hop count can be explained as in Figure 4.

Quality of Service (QoS) defines the ability to provide services and defines the characteristics and characteristics of a service. Parameters of QoS is one benchmark indicator of the quality of service from the computer network itself. The QoS standard used refers to the International Telecommunication Union Telecommunication (ITU-T) standard. ITU-T is part of ITU which is an international organization established to standardize, regulate and standardize international radio and international telecommunications. Quality of Service (QoS) has a flow of data packets that are used as measuring parameters of Service Internet Protocol Network: reliability; packet loss, bandwidth; and throughput.

\section{Method}

The topology used in this study is shown in Figure 5, on the topology there is one client laptop, one server, UTP cable, and six router units. The client and server laptops use IPv6 and are connected over IPv4 networks. The client and server laptops are connected by routers running dynamic routing protocol OSPFv3 or RIPng.

QoS testing on OSPFv3 and RIPng protocols is performed with three scenarios in each routing protocol, with the following details

1. OSPFv3 with best active path conditions

2. OSPFv3 when network changes

3. OSPFv3 with best path down comdition (Stable)

4. RIPng with the best active path conditions

5. RIPng when network changes

6. RIPng with best down condition (Stable)

Testing is done by testing the QoS parameters when the client computer downloads files with a 100 Mega Byte .iso extension for File1.iso, 300 Mega Bytes for File2.iso, 500 Mega Bytes for File3.iso, 700 Mega Bytes for File4.iso, and 1 Giga Byte for File5.iso from server. Variable QoS tested on each file consists of three parameters, namely the parameter delay, packet loss, and throughput. QoS testing is done by capturing the packet using wire shark tool, then calculation of the communication that occurs between the client and server to obtain the average value of delay and packet loss.

\section{Result and Discussion}

\subsection{Testing of Topology}

In QoS Analysis on OSPFv3 and RIPng Using GRE Tunnel Using Mikrotik Router is used 6 routers. In OSPFv3 topology, the six routers are divided into two separate backbone areas over the Internet using the IPv4 network. In the RIPng topology, the six routers are also 
divided into two sites, namely site A and site B. As for one area or site there is one client computer that will access the server located on another area or site. Topology testing is shown in figure 5 .

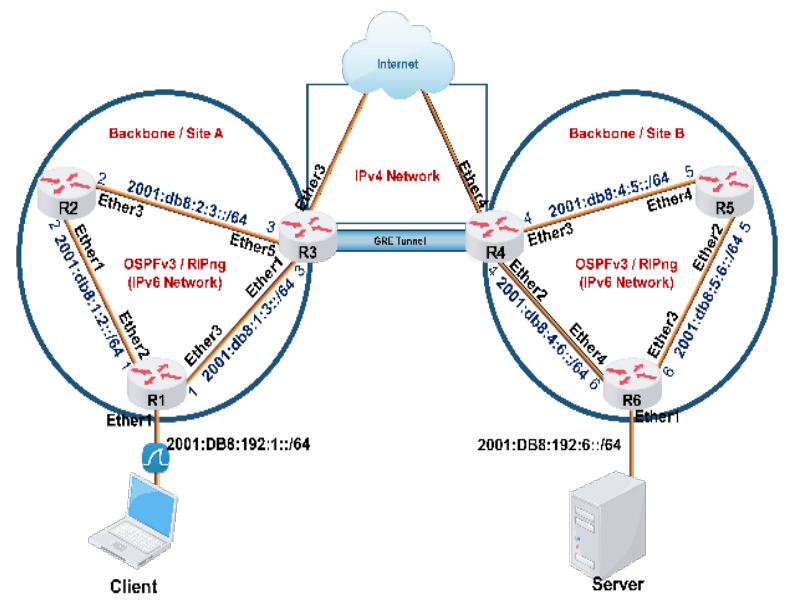

Fig.5. Topology Design

\subsection{Average QoS Comparison}

After obtaining the average results of three QoS tests on OSPFv3 and RIPng using GRE Tunnel, then the average table is displayed in the form of bar graph. The bar graph is displayed so that the QoS differences in each of the routing protocols are more clearly visible. Graphs are displayed in three views, ie. QoS charts on active best path conditions, QoS charts in the event of network changes due to the main down path, and QoS graphs in stable condition after the best path down.

Based on the figure 7 , it appears that the average delay on OSPFv3 is better than RIPng for the best active path conditions.

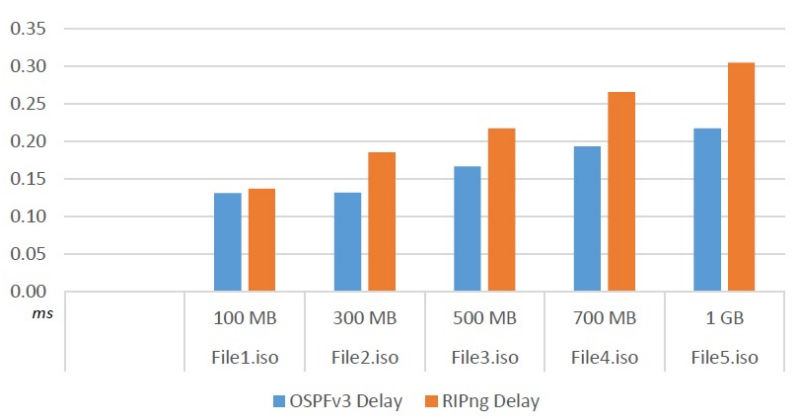

Fig.7. Chart of Comparison Delay Best Active Path Condition

In figure 8 , OSPFv3 is better than RIPng based on the average packet loss on testing with the best active path conditions. In the packet loss parameter, it is found that the packet loss value in the initial frame of the download process is greater than the packet loss in the next frame, in other words the packet loss value continues to decrease in the next frame, thus the higher frame number will be the less number of packet loss produced. This applies to all test scenarios. While the number of frame number depends on the size of the file is transmitted. The larger the size of the file being transmitted, the more frames are transmitted, and the higher the frame number obtained. Figure 9 shows that the average throughput of OSPFv3 is better than RIPng on all tests.

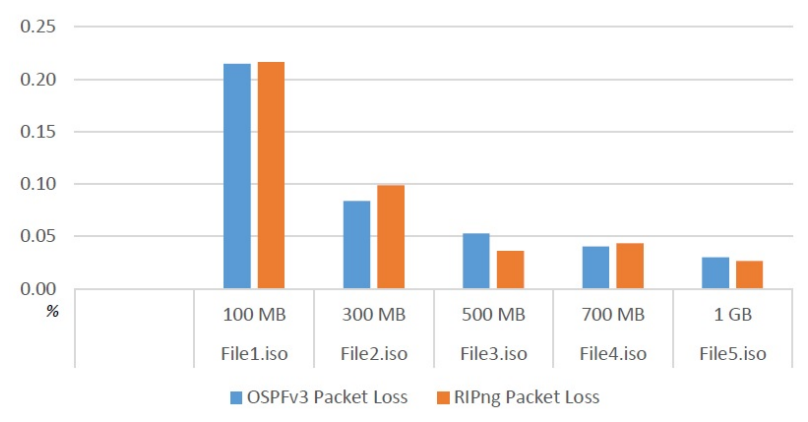

Fig. 8. Chart of Comparison Packet loss Best Active Path Conditon

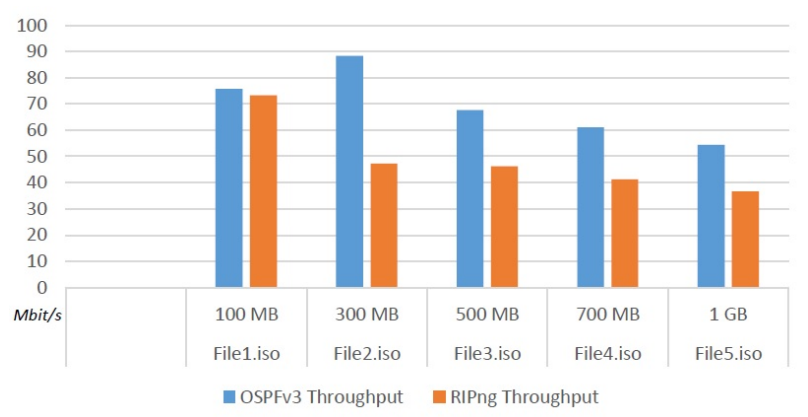

Fig.9. Chart of Comparison Throughput Best Active Path Condition

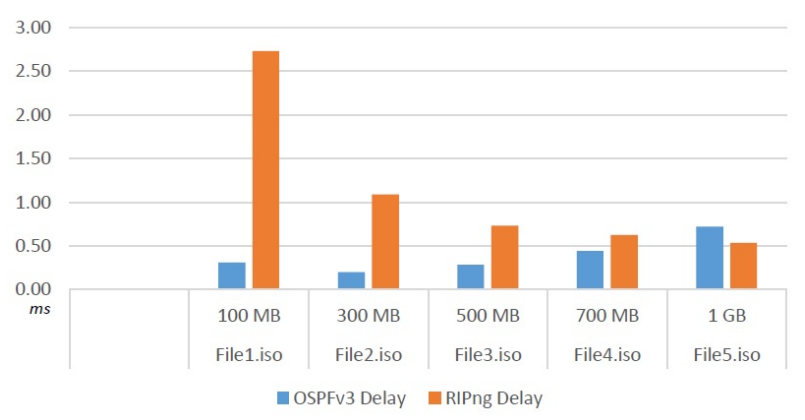

Fig.10. Chart of Comparison Delay Network Changes Condition

Figure 10 shows that overall delay in OSPFv3 is better than RIPng on network change conditions. The average delay in the RIPng routing protocol for network change scenarios is different from normal conditions, this is affected by the time it takes to update the routing table after the main path down until the file download process runs again and is influenced by the incremental number of frames from file1.iso to file5.iso. Where, the greater the load of transmitted files will be more and more frames are sent which is then calculated with the time required for the transmission of the file including 
the time required to update the routing table until the download process file runs back which then obtained the average value of delay. Figure 11 shows that the average packet loss in OSPFv3 is better than RIPng on all tests under network change conditions.

In Figure 12, it is seen that the mean throughput of OSPFv3 is better than RIPng based on the test sample on each test. The throughput in RIPng becomes different from the normal conditions, where the larger the load file size the larger the throughput will be. This is due to the effect of the time required to update the routing table after the best path down until the file download process runs again, and is affected by the incremental byte number from file1.iso to file5.iso, where the greater the load of files transmitted will be larger in size bytes which are then calculated with the time required for the transmission of the files to obtain throughput.

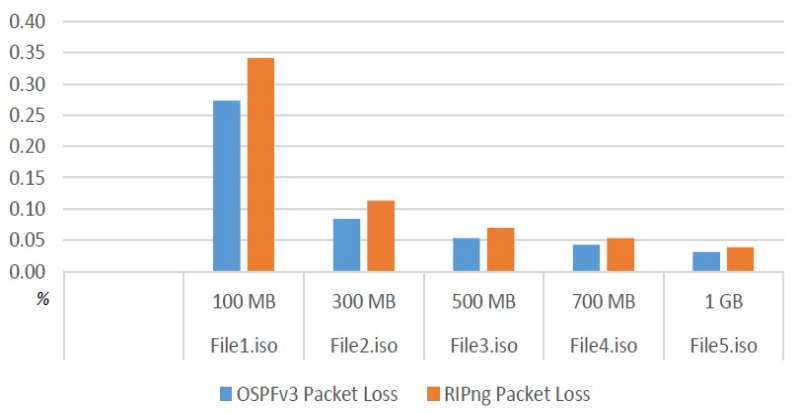

Fig.11. Chart of Comparison Packet loss Network Changes Condition

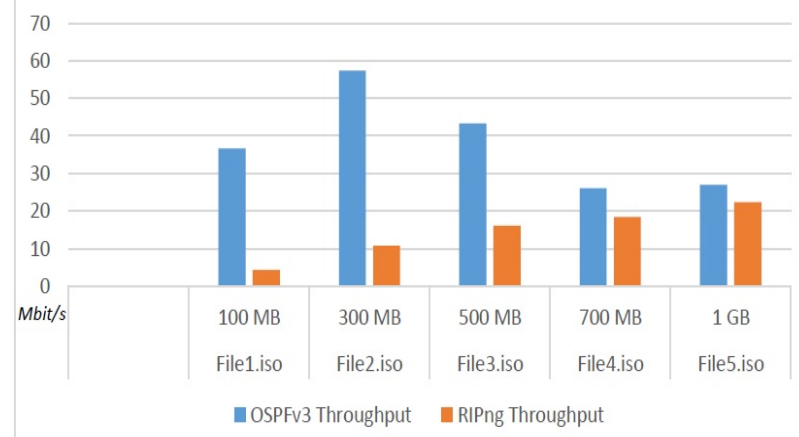

Fig.12. Chart of Comparison Throughput Network Changes Condition

Comparison of QoS in OSPFv3 and RIPng for stable network conditions after network changes so that the best paths are also displayed in the form of bar graphs. Based on the delay, packet loss, and throughput parameter are shown in figure 13,14, and 15.

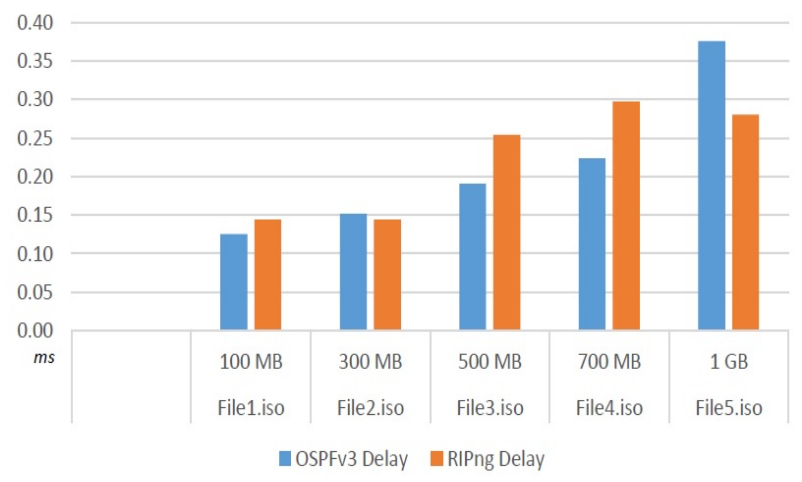

Fig.13. Chart of Comparison Delay Best Path Down Condition

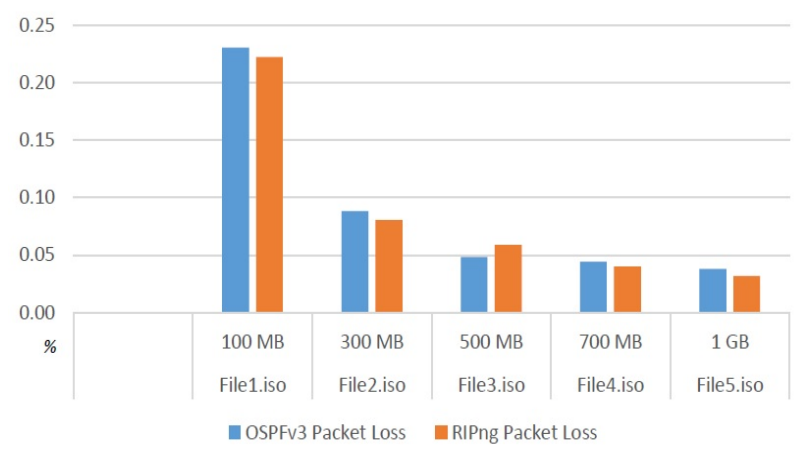

Fig. 14. Chart of Comparison Packet loss Best Path Down Condition

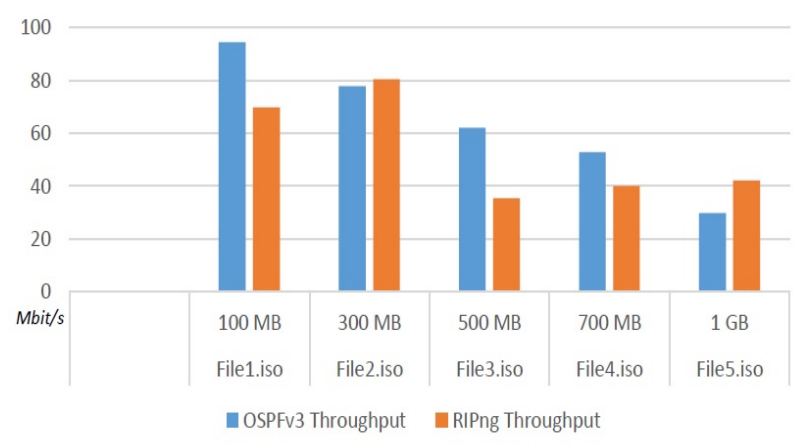

Fig.15. Chart of Comparison Throughput Best Path Down Condition

Based on the above test, the average value of delay, packet loss, and throughput of both routing protocols in all those scenarios are included in the good category based on ITU-T G.114 standard.

\section{Conclusion and Recommendation}

Based on QoS testing of OSPFv3 and RIPng protocol method using GRE tunnel, it is found that overall OSPFv3 is better than RIPng on delay, packet loss, and throughput parameters in all scenarios. In addition, the results obtained that the data load will affect the delay in other words the greater the size of the downloaded file, the greater the average delay obtained, except in the RIPng routing protocol for network change scenarios, this is because RIPng takes a longer time than OSPFv3 
in updating the routing table until the download process runs again which is also the effect of the characteristics of each type of protocol, and also influenced by the number of incremental frames from file1.iso to file5.iso sent by the server to the client when the download process .

The characteristics of each protocol affect the results obtained, so for similar research it is advisable to analyze other routing protocols that are included in the Interior Gateway Protocol (IGP) and Enhanced Interior Gateway Routing Protocol (EIGRP) with RIPng for distance vector protocol, and OSPFv3 with Intermediate System to Intermediate System (IS-IS) on the type of link state protocol for IPv6 networks. It is also recommended to test the characteristics, QoS, and performance of each of these routing protocols against various other experimental scenarios.

\section{References}

1. Masruroh, Situ Ummi., Robby, Fadly., Hakiem, Nashrul., Informatics and Computing (ICIC), (2016)

2. Nixon, Dr. J Sebastian, Dr A Francis Saviour Devaraj., International Journal of Computer Science and Information Technology \& Security (IJCSITS), $\mathbf{5}, 6,(2015)$

3. Narula, Rajneesh., Aggarwal, Pallavi., International Journal of Technical Research and Applications, 2, 6 (2014)

4. Hanumanthappa, International Journal of Computer Science and Information Security (IJCSIS), 3, 1 (2009)

5. Saraj, Tariq., Hanan, Abdul., Akbar, M. S., Yousaf, M., Qayyum, A., dan Tufail, M. (2015, December). Information Assurance and Cyber Security (CIACS), (2015)

6. Juanda, Dadan Hendra, Analisa Perbandingan Metode Protokol Routing Distance Vector Dengan Protokol Routing Link State Terhadap Aplikasi Layanan Paket Data Real Time Menggunakan Router Mikrotik. (Institut Teknologi Padang, Padang, 2016)

7. Reddy, M. P. R., Krishna, M. D. V., Madhavi, M. S. P., Reddy, M. B. R., dan Reddy, M. D. M. the future configuration version protocals of global and private ip-ipv6 securit featurs.Int. Journal of research Science \& Management. (2014)

8. Komarudin, Ahmad Rosid., Pranozal, Alex., Feriansyah., dan Irpan, Teori dan Konsep OSPF di Cisco dan MikroTik. ID-Networkers, Jakarta, (2016) 